\title{
Capital Adjustment and Limit Cycles: An Empirical Analysis Based on the Threshold Autoregressive Model
}

\author{
Yasuyuki Nishigaki ${ }^{1}$, Daiki Maki ${ }^{1}$, Mitsuhiko Satake ${ }^{2}$ \\ ${ }^{1}$ Faculty of Economics, Ryukoku University, Kyoto, Japan \\ ${ }^{2}$ Faculty of Economics, Doshisha University, Kyoto, Japan \\ Email address: \\ nisigaki@econ.ryukoku.ac.jp (Y. Nishigaki), maki@econ.ryukoku.ac.jp (D. Maki), msatake@mail.doshisha.ac.jp (M. Satake)
}

\section{To cite this article:}

Yasuyuki Nishigaki, Daiki Maki, Mitsuhiko Satake. Capital Adjustment and Limit Cycles: An Empirical Analysis Based on the Threshold Autoregressive Model. International Journal of Economic Behavior and Organization. Special Issue: Recent Developments of Economic Theory and Its Applications. Vol. 3, No. 2-1, 2015, pp. 52-59. doi: 10.11648/j.ijebo.s.2015030201.19

\begin{abstract}
In this study, we investigate the non-linearity of the Japanese business cycle based on the theoretical concept of the limit cycle. To analyze the time series of capital stock and GDP simultaneously based on the theoretical relationships predicted by the limit cycle, we incorporate the capital coefficient into a Kaldor-type dynamic model and apply the threshold autoregressive (TAR) model to it to investigate fluctuations in the coefficient that are concurrent to the underlying oscillation of the limit cycle. The estimation results indicate that these time series are subject to the three-regime TAR model and that the middle regime has divergence and the outside regimes have convergence, suggesting that the process has a non-linear phenomenon typically caused by limit cycles.
\end{abstract}

Keywords: Business Cycle, Limit Cycle, Capital Coefficient, Threshold Autoregressive Model

\section{Introduction}

In endogenous business cycle theory, many studies have utilized non-linear economic dynamics such as chaotic dynamics and limit cycle theory to model business cycles. These authors have succeeded in explaining the seemingly irregular fluctuations in actual time series. The basic idea of utilizing limit cycles in business cycle theory was first demonstrated by Kaldor (1940) and later mathematically formulated by Chang and Smith (1971) and Varian (1979). Since then, the theoretical interest of economists has expanded this literature (e.g., Lorentz (1993)).

Concurrent with the expansion of the theoretical investigation, empirical interest has investigated the existence of non-linearity in actual macro-economic time series data. Tong and Lim (1980) and Tong (1983) indicated that several non-linearities including the limit cycle in macro-economic time series are explained by the threshold autoregressive (TAR) model. Potter (1995) and Koop and Potter (1999) applied the TAR model to GDP and macro-economic time series data from the United States and United Kingdom, while Kraeger and Kugler (1993) and Sarantis (1999) applied it to the time series of the exchange rates between the United States dollar and Japanese yen as well as the United States dollar and
Euro.

In this study, we apply the TAR model to investigate the non-linearity of the Japanese business cycle based on the theoretical concept of the limit cycle introduced by Kaldor (1940). Kaldor constituted a business cycle model characterized by a two-dimensional limit cycle by introducing a sigmoid-shaped investment function based on the profit principle of investment. In a limit cycle, national income (or GDP) and the level of capital stock are inter-related in the closed orbit and continuously oscillating in the two-dimensional space. Therefore, in statistical analysis, both time series should be analyzed simultaneously based on the causal relationships predicted by the theory.

However, while non-linearity tests utilizing the TAR model have been conducted on a single time series, to our knowledge, the analysis has remained one-dimensional thus far. To address this methodological limitation, we introduce a new variable into the Kaldor model, the so-called capital coefficient, and consider the process of capital adjustment. By applying the TAR model to this coefficient, we investigate the fluctuations in the coefficient concurrent to the underlying oscillation of the limit cycle.

For the empirical investigation, we specifically address the connotations of limit cycles predicted by continuous time theoretical models and the macro-economic time series 
presented in discrete time periods by investigating the dynamic consequences of a discrete time version of the model. Bischi et al. (2001) and Agliari et al. (2007) introduced a discrete time Kaldor-type business cycle model and investigated interesting phenomena unexpected from continuous time dynamics. According to their investigations, we consider the implications of applying theoretical consequences predicted by a continuous time model to time series data.

The rest of the paper is organized as follows. In section 2, a Kaldor-type dynamic model is presented and the conditions for the existence of the limit cycle are examined. In section 3, a discrete time version of a Kaldor-type model is introduced and the concept of limit cycles in discrete time is explained. In section 4, we introduce the capital coefficient for the preparation of the empirical study. The method of the empirical analysis (applying the TAR model) and its results are presented in sections 5 and 6 , respectively. Section 7 concludes.

\section{Kaldor's Business Cycle Model and Limit Cycles}

Let us consider a simple dynamic national income adjustment model:

$$
\begin{gathered}
\dot{Y}=\alpha[I(Y, K)-S(Y, K)], \\
\dot{K}=I(Y, K)-\delta K,
\end{gathered}
$$

where $Y$ is (gross) national income, $K$ is capital stock, $I(Y, K)$ is the (gross) investment function, and $S(Y, K)$ is the saving function. The parameter $\alpha$ is a constant adjustment variable and $\delta$ is the depreciation rate of capital.

The investment function that Kaldor incorporated into his model is a sigmoid-shaped function based on the profit principle of investment. This non-linear function depends on both the level of national income and the level of capital stock.

Investment $I$ is assumed to be an increasing function of national income because an increase in national income, under a given level of capital stock, increases the sales of products and profits of firms, which generate an incentive to invest. However, the marginal propensity to invest decreases if the actual income level diverges from its equilibrium level. In the case of higher current income, higher investment cost and anticipation of the end of the boom reduce the investment incentive, whereas the level of production can rise without increasing investment because of the existence of unused capital stock in the case of lower national income.

Furthermore, investment is assumed to be a decreasing function of capital stock because the induced investment effect of national income does not occur in the presence of unused capital stock. The investment function of this type, therefore, adjusts current capital stock to its normal level, which means that as the level of current capital stock $K$ increases, the investment function shifts downward.

Derivative representations of the properties of these assumptions on the investment function, in sum, are $I_{Y}>0, I_{K}<0$ and for the certain level of the national income $Y^{*} 1$, if actual national income is less than that level (i.e., $Y<Y^{*}$ ), then $I_{Y Y}>0$; on the contrary, if actual national income is larger than that level (i.e., $Y>Y^{*}$ ), then $I_{Y Y}<0$.

For the saving function, it is assumed that saving is a linear increasing function of national income as well as an increasing function of capital stock. Derivative expressions of the properties of these assumptions are $0<S_{Y}<1, S_{K}>0$.

A fixed point of the system is a solution that satisfies $\dot{Y}=\dot{K}=0$ in equations (1) and (2).

$$
\begin{array}{r}
I(Y, K)-S(Y, K)=0, \\
I(Y, K)-\delta K=0 .
\end{array}
$$

We examine the properties of the solution in the system of equations (3) and (4) by applying the Poincare-Bendixson theorem (see Appendix A). Here, if the solution locus of the system is characterized by limit cycles, the level of national income and capital stock are continuously fluctuating, implying the existence of long-lasting business cycles.

The procedure for applying the Poincare-Bendixson theorem is as follows2. First, the existence of a stable point with saddle point stability is excluded. A condition that satisfies the unstable nature of the stable point is then examined. Finally, the phase of the system of differential equations and existence of compact and invariable set " $D$ " are examined. As a more precise investigation is described in Appendix A, only the results are presented here.

To exclude the possibility of the saddle point stability of the fixed point(s), $I_{Y}^{*}-S_{Y}^{*}>0$ is required in the neighborhood of the fixed point. Furthermore, for the instability of the fixed point $\alpha\left(I_{Y}^{*}-S_{Y}^{*}\right)>-\left(I_{K}^{*}-\delta\right)$. From the assumptions mentioned above, $I_{K}<0$, which makes the right-hand side of the equation positive. The national income-induced investment effects $I_{Y}$, therefore, should be considerably higher3.

In Kaldor's model, a set $D=\left\{(Y, K) \mid 0 \leq Y \leq Y_{1}, 0 \leq K \leq K_{1}\right\} \quad$ is $\quad$ compact according to the assumptions and the vector space is inward

\footnotetext{
1 We assume that the fixed point $Y^{*}$ is sufficiently smaller than the level of full employment income in order to secure the fluctuations of national income.

2 For a detailed analysis, see Appendix A.

3 Based on the assumption concerning the non-linear investment function stated above, $I_{Y}$ decreases as $Y$ moves far enough from the stable point, which makes $\left(I_{Y}-S_{Y}\right)$ negative when the level of national income is small or large enough. The existence of closed orbits, therefore, is not excluded.
} 
pointed at the boundary of the set. Figure 1 shows the typical phase diagram and " $\mathrm{D}$ " set of the Kaldor model.

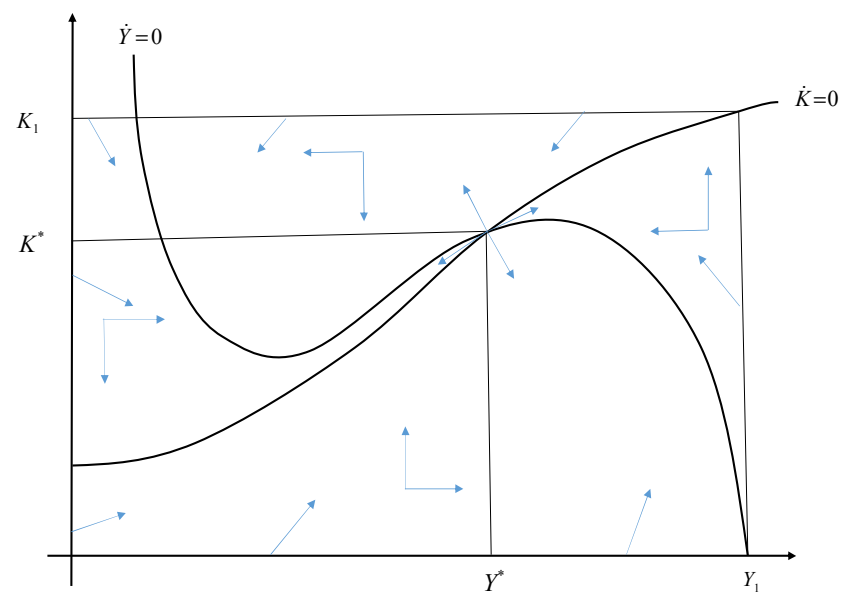

Figure 1. Phase and "D” set of the Kaldor model.

\section{Limit Cycles in Discrete Time}

In the following sections of statistical investigation, we utilize a time series data set, which implies that the dynamic model underlying the investigation is supposed to be a discrete time model. A limit cycle in discrete time is defined as follows see Tong and Lim (1980).

Let $x_{n}$ denote a $k \times 1$ dimensional state that satisfies the equation

$$
x_{n}=f\left(x_{n-1}\right) \text {. }
$$

A limit point in the vector space is defined as follows. A $k \times 1$ dimensional vector $x^{*}$ is called a limit point if there exists $x_{0} \neq x^{*}$ such that, starting with $n=0, x_{n}$ tends to $x^{*}$ as $n$ tends to infinity: $\lim _{n \rightarrow+\infty} x_{n}=x^{*}$.

A limit cycle in discrete time is defined as follows. Let $C$ denote the set of $k \times 1$ dimensional vector $c_{i}$, $i=1, \cdots, T, T$ being a positive integer $\leq \infty$.

1) $C$ is called a limit cycle of period $T$ if there exists $x_{0} \notin C$, such that as $n \rightarrow+\infty, x_{n}$ ultimately falls to $x^{*}$ : $x_{n} \rightarrow x^{*} \quad$ 2) $\quad c_{i}=f\left(c_{i-1}\right) i=2,3, \ldots, T$, $c_{T+i}=c_{i} \quad i=1,2, \ldots, T$, and 3) $T$ is the smallest such positive integer.

The discrete time version of the Kaldor model that appeared in Bischi et al. (2001) and Agliari et al. (2007) is

$$
\begin{gathered}
Y_{t+1}-Y_{t}=\alpha\left[I_{t}\left(Y_{t}, K_{t}\right)-S_{t}\left(Y_{t}\right)\right], \\
K_{t+1}-K_{t}=I_{t}\left(Y_{t}, K_{t}\right)-\delta K_{t},
\end{gathered}
$$

where the suffix $t$ represents the time period. The saving and investment functions are further assumed by using the following fixed coefficients:

$$
S_{t}\left(Y_{t}\right)=\sigma Y_{t}
$$

$$
I_{t}\left(Y_{t}, K_{t}\right)=\sigma \mu+\beta\left(\frac{\sigma \mu}{\delta}-K_{t}\right)+\arctan \left(Y_{t}-\mu\right)
$$

where $\sigma$ represents the propensity to save $(0<\sigma<1), \mu$ is expected income $\left(Y_{t}^{e}=\mu\right)$, and $\sigma \mu / \delta$ is the "normal" level of capital stock.

In equation (9), two components are considered; the first one is proportional to the difference between "normal" capital stock and current stock, according to the adjustment coefficient $\beta$; the second one is an increasing and non-linear function of the difference between current income and its "normal" level. By using these two components, equation (9) generates the basic properties of the investment function of the profit principle.

By substituting these two functions into the dynamic model (6) and (7), we obtain the two-dimensional difference equations of the non-linear dynamic model:

$$
\begin{gathered}
Y_{t+1}-Y_{t}=\alpha\left[\sigma \mu+\beta\left(\frac{\sigma \mu}{\delta}-K_{t}\right)+\arctan \left(Y_{t}-\mu\right)-\sigma Y_{t}\right] \\
K_{t+1}-K_{t}=\sigma \mu+\beta\left(\frac{\sigma \mu}{\delta}-K_{t}\right)+\arctan \left(Y_{t}-\mu\right)-\delta K_{t}
\end{gathered}
$$

The fixed points of the dynamic model are obtained by setting equations (10) and (11), with $Y_{t+1}-Y_{t}=0$ and $K_{t+1}-K_{t}=0$ :

$$
K=\frac{\sigma}{\delta} Y
$$

$$
\sigma\left(1+\frac{\beta}{\delta}\right)(Y-\mu)=\arctan (Y-\mu)
$$

Bischi et al. (2001) examined the uniqueness and stability of the fixed point $(\mu, \mu \cdot \sigma / \delta)$ on these systems of equations and showed that the fixed point is unique if $1>\sigma \geq \delta /(\delta+\beta)$, while if $\alpha \geq(\delta+\beta)^{2} / \beta$, Hopf bifurcation occurs and stable oscillations along a limit cycle 
are generated 4.

\section{Capital Coefficient and Capital Adjustment in the Kaldor Model}

By introducing the capital coefficient' $K / Y$ to the Kaldor-type dynamic model, the two-dimensional oscillations of the limit cycle are indicated by fluctuations in the coefficient in a single dimension. Figure 2 shows the relations between fluctuations in the capital coefficient $K / Y$ and underlying oscillations of the limit cycles in the $(K, Y)$ space.

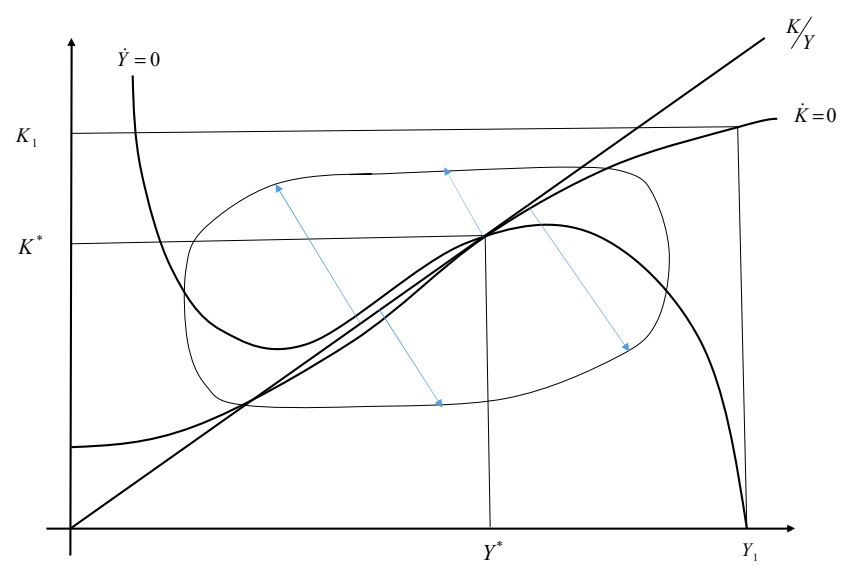

Figure 2. Capital coefficient and limit cycle.

The assumptions on the investment function mentioned above mean that an increase or decrease in investment shifts capital stock to the level that ensures a certain capital operation rate, which is determined by reflecting the "normal" level of national income. Continuous fluctuations in the capital coefficient are thus generated in the Kaldor model from the underlying limit cycle in the $K-Y$ space.

\section{Method of the Empirical Analysis}

As an assumption of an empirical analysis of a limit cycle, let $z_{t}$ be an observable variable. We consider the data generating process (DGP) as follows:

$$
z_{t}=a^{\prime} d_{t}+u_{t}
$$

where $a^{\prime} d_{t}$ represents the deterministic components. When the data have a nonzero mean ( $a=a_{0}$ and $\left.d_{t}=1\right)$, the demeaned data are used. When the data have a nonzero mean and a trend $\left(a=\left[\begin{array}{ll}a_{0} & a_{1}\end{array}\right]^{\prime}, d_{t}=\left[\begin{array}{ll}1 & \mathrm{t}\end{array}\right]^{\prime}\right)$, we use the demeaned and detrended data. $u_{t}$ follows the three-regime TAR process:

$$
u_{t}=\left\{\begin{array}{c}
\varphi_{1} u_{t-1}+e_{t}, \text { if } u_{t-1} \leq \gamma_{1} \\
\varphi_{2} u_{t-1}+e_{t}, \text { if } \gamma_{1}<u_{t-1} \leq \gamma_{2}, \\
\varphi_{3} u_{t-1}+e_{t}, \text { if } u_{t-1}>\gamma_{2}
\end{array}\right.
$$

where $e_{t}$ is a zero mean error and $\gamma_{1}$ and $\gamma_{2}$ are thresholds.

4 See Bischi et al. (2001).
We assume $\gamma_{1}<\gamma_{2}$. The existence of the stationarity of $u_{t}$ requires that $-1<\varphi_{1}<1$ and $-1<\varphi_{3}<1$. The stationary three-regime TAR process has the feature such that $u_{t}$ moves toward 0 when $u_{t-1} \leq \gamma_{1}$ and $u_{t-1}>\gamma_{2}$ even if $\varphi_{2}>1$ or $\varphi_{2}<-1$ in the middle regime.

For example, we consider the process with $\varphi_{1}=\varphi_{3}=0.5$, $\varphi_{2}=1.05, \gamma_{1}=-2$, and $\gamma_{2}=2$. When we set the error term to $0, u_{t}$ and $u_{t-1}$ draws the phase diagram displayed in Figure 3. From this diagram, it is evident that the origin is only one and unstable fixed point. When the process is in the outside regime, it moves to the middle regime. When the process is in the middle regime, it gradually moves to the outside regime. Accordingly, the relationship between $u_{t}$ and $u_{t-1}$ has two period cycles typically characterized by a limit cycle.

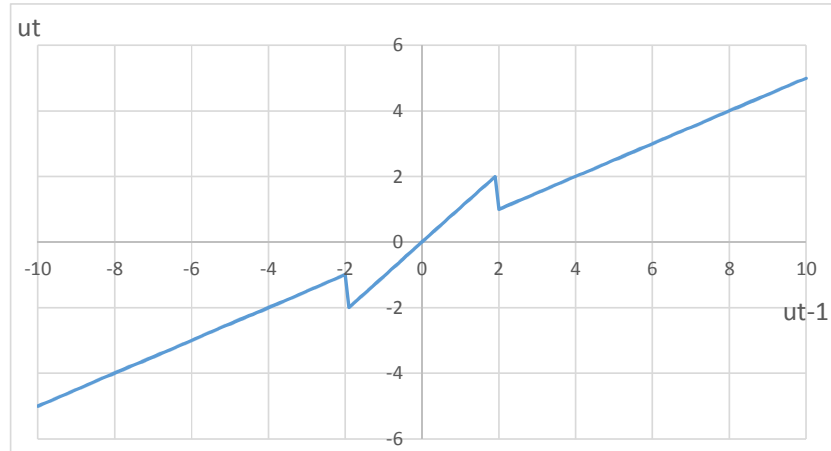

Figure 3. The phase diagram of $u_{t}$ and $u_{t-1}$.

However, we have to test for the stationarity of the process before we estimate the TAR model. We first test for a unit root against the three-regime TAR process. If a process has a unit root, it does not have a limit cycle because the process is divergent and does not display cyclical behavior. On the contrary, if the process is characterized by the three-regime TAR one, especially with the divergent middle regime $\varphi_{2}>1$ or $\varphi_{2}<-1$, the process has cyclical behavior inside and outside the thresholds, as shown in Figure 3.

To test for a unit root, we consider the following regression model:

$$
\begin{gathered}
\Delta u_{t}=\rho_{1} u_{t-1} I\left\{u_{t-1} \leq \gamma_{1}\right\}+\rho_{2} u_{t-1} I\left\{\gamma_{1}<u_{t-1} \leq \gamma_{2}\right\}+ \\
\rho_{3} u_{t-1} I\left\{u_{t-1}>\gamma_{2}\right\}+\sum_{j=1}^{p} b_{j} \Delta u_{t-p}+\varepsilon_{t},
\end{gathered}
$$

where $I\{\cdot\}$ is the indicator function such that $I\{\cdot\}$ is 1 if $\{\cdot\}$ is true and 0 otherwise, and $\varepsilon_{t}$ is a zero mean error. We test for the null hypothesis of a unit root with $\rho_{1}=\rho_{2}=\rho_{3}=0$. Similar tests are those of Bec et al. (2004), Kapetanios and Shin (2006), and Park and Shintani (2014). As shown by these authors, the tests based on a three-regime TAR model have a better power for the process compared with standard tests. In particular, the tests of Kapetanios and Shin (2006) and Park and Shintani (2014) restrict the middle regime to $\varphi_{2}=1$. Moreover, while the test of Bec et al. (2004) allows for different intercepts and lag parameters in each regime, our model is based on the tests introduced by Kapetanios and Shin (2006) and Park and Shintani (2014), but it allows for $\varphi_{2}>1$ in the middle regime. 


$$
\begin{gathered}
\underset{\Delta u_{t-p}^{p}}{\text { By }}=\left(\Delta u_{t-1}, \cdots, \Delta u_{t-p}\right)^{\prime},(16) \text { is rewritten as } \\
\Delta u_{t}=U_{t}^{\prime} \theta+\varepsilon_{t},
\end{gathered}
$$

where $\quad U_{t}=\left(u_{t-1} I\left\{u_{t-1} \leq \gamma_{1}\right\}, u_{t-1} I\left\{\gamma_{1}<u_{t-1} \leq\right.\right.$ $\left.\left.\gamma_{2}\right\}, u_{t-1} I\left\{u_{t-1}>\gamma_{2}\right\}, \Delta u_{t-p}^{\prime}\right)^{\prime}$ and $\theta=\left(\rho_{1}, \rho_{2}, \rho_{3}, b^{\prime}\right)^{\prime}$.

Let $\hat{\theta}$ be the OLS estimator of $\theta$ and $\hat{\varepsilon}_{t}$ be the OLS residual of $\varepsilon_{t}$ in (17). When thresholds are given, the statistic to test for the null hypothesis is given by

$$
\mathrm{W}(\gamma)=\frac{1}{\hat{\sigma}^{2}} \hat{\rho}^{\prime}\left[R\left(\sum_{t=1}^{T} U_{t} U_{t}^{\prime}\right)^{-1} R^{\prime}\right]^{-1} \hat{\rho},
$$

where $\hat{\rho}=\left(\hat{\rho}_{1}, \hat{\rho}_{2}, \hat{\rho}_{3}\right)^{\prime}$ is the OLS estimator of $\rho$ and $\mathrm{R}$ is the $3 \times(p+3)$ matrix such that $\mathrm{R} \hat{\theta}=\hat{\rho}$. For unknown thresholds, we compute (18) for each possible threshold and take the largest value across all possible thresholds. Then, the following supremum-type statistic is used:

$$
\sup W(\gamma)=\sup _{\gamma \in\left[\gamma_{\min }, \gamma_{\max }\right]} W(\gamma) .
$$

When we employ (19), we first arrange the values of $u_{t}$ in ascending order. Second, we select $\gamma_{\min }=u_{([5 T / 100])}$ and $\gamma_{\text {max }}=u_{([95 T / 100])}$, where [ ] is the integer part. Furthermore, we allow for the existence of at least $10 \%$ of the observations for the inside and outside regimes. For the setting, $\gamma_{1}$ includes equally spaced 100 points between the $5 \%$ and $45 \%$ quantiles of the arranged values and $\gamma_{2}$ includes equally spaced 100 points between the $55 \%$ and $95 \%$ quantiles. This test is based on Maki (2009) and Maki and Kitasaka (2014).

Table 1 shows the critical values of the tests. The asymptotic critical values approximated by $T=1,000$ are obtained from 10,000 replications. We present three models: Model 0 contains no deterministic terms; Model 1 contains an intercept in the DGP; and Model 2 contains both an intercept and a trend in the DGP.

Table 1. Critical values

\begin{tabular}{llll}
\hline & $\mathbf{1 0 \%}$ & $\mathbf{5 \%}$ & $\mathbf{1 \%}$ \\
\hline Model 0 & 17.19 & 19.39 & 24.24 \\
Model 1 & 17.54 & 19.57 & 24.37 \\
Model 2 & 19.35 & 21.69 & 26.58 \\
\hline
\end{tabular}

\section{Empirical Evidence}

We investigate whether $K / Y$ has a limit cycle, where $K$ and $Y$ represent capital stock and GDP, respectively. We use private capital stock and real GDP as $K$ and $Y$, respectively, and employ seasonally adjusted data both in level and in logarithm. The quarterly data obtained from Nikkei Needs consist of 176 observations from 1970Q1 to 2013Q4. We employ Model 2 to determine the maximum lag length for $\mathrm{p}=12$ and then exclude the insignificant augmentation terms until the last term is significant.

Table 2 presents the results of the unit root tests. We use heteroskedastic-robust test statistics. The table shows that test (19) rejects the null hypothesis of a unit root at the 5\% significance level, whereas the standard Dickey-Fuller-type test does not. These results provide strong evidence that both level and log data have a TAR process.

Table 2. Unit root test results.

\begin{tabular}{lll}
\hline & TAR & ADF \\
\hline Level & $25.09(* *)$ & -2.36 \\
Log & $25.21(* *)$ & -2.647 \\
\hline
\end{tabular}

$(* *)$ indicates the $5 \%$ significance level.

Table 3 presents the estimation results of the TAR model (16). The thresholds are estimated by minimizing the sum of the squared residuals over $\left[\gamma_{\min }, \gamma_{\max }\right]$, where $\gamma_{\min }$ and $\gamma_{\max }$ are set to the $5 \%$ and $95 \%$ quantiles of the residuals, respectively. For the level data, the estimates of the outer regime parameters $\rho_{1}$ and $\rho_{3}$ are -0.051 and -0.048 , respectively. From the estimates, the DGP satisfies the stationary condition. Additionally, the estimate of the middle regime parameter $\rho_{2}$ is 0.015 . This finding indicates that the middle regime has divergence and the outside regimes have convergence. We obtain similar results for the log data. For both types of data, the estimates of $\rho_{1}$ and $\rho_{3}$ satisfy the stationary condition and the estimate of $\rho_{2}$ is larger than zero. Therefore, the results are consistent with the feature of non-linear phenomena including limit cycles described in section 5 .

Table 3. TAR estimation results.

\begin{tabular}{llllllll}
\hline & $\boldsymbol{\rho}_{\mathbf{1}}$ & $\boldsymbol{\rho}_{\mathbf{2}}$ & $\boldsymbol{\rho}_{\mathbf{3}}$ & $\boldsymbol{\gamma}_{\mathbf{1}}$ & $\boldsymbol{\gamma}_{\mathbf{2}}$ & $\mathbf{s}$ & MR\% \\
\hline Level & -0.051 & 0.015 & -0.048 & -8.159 & 12.10 & 0.813 & 51.70 \\
& $(0.017)$ & $(0.009)$ & $(0.013)$ & & & & \\
Log & -0.052 & 0.013 & -0.048 & -0.102 & 0.139 & 0.010 & 53.97 \\
& $(0.020)$ & $(0.008)$ & $(0.012)$ & & & & \\
\hline
\end{tabular}

Heteroskedastic-robust standard errors are given in parentheses. s represents the standard deviation of the error term. MR\% denotes the percentage of observations in the middle regime.

The difference equation system without stochastic terms is derived from the empirical results for the level data on table 3 as5,

\footnotetext{
5 The authors are largely indebted to the referee comments for the following investigations. They wish to thank again anonymous referees of this journal.
}

$$
\begin{aligned}
& \text { (1) } u_{t-1} \leq-8.159 \quad \Rightarrow u_{t}=0.949 u_{t-1} \\
& \text { (2) }-8.159<\mathrm{u}_{t-1} \leq 12.10 \Rightarrow u_{t}=1.015 u_{t-1} . \\
& \text { (3) } u_{t-1}>12.10 \quad \Rightarrow u_{t}=0.952 u_{t-1}
\end{aligned}
$$

Based on this system of equations, we can draw the following phase diagram: 


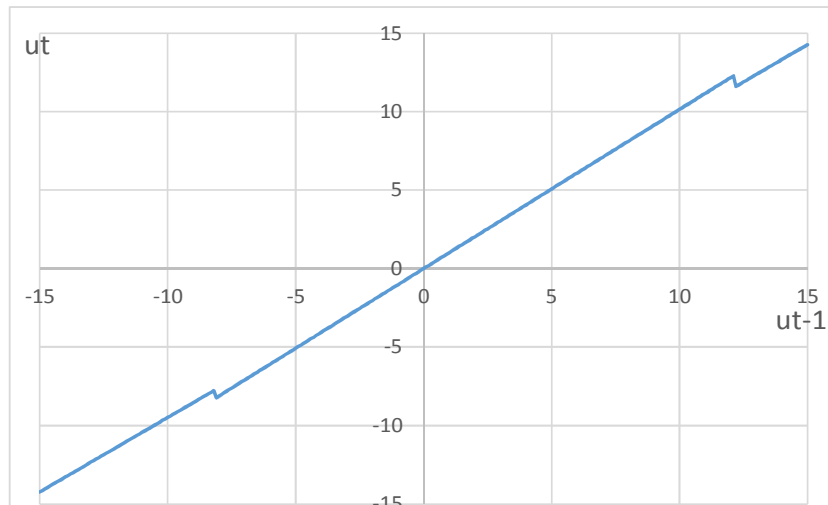

Figure 4. The phase diagram of the estimated difference equation system

It is obvious that the fundamental dynamics of the phase diagram is the same as that of figure 3. By implementing the simulation method on the difference equation system with the starting point of 12.1 , the following diagram is obtained.

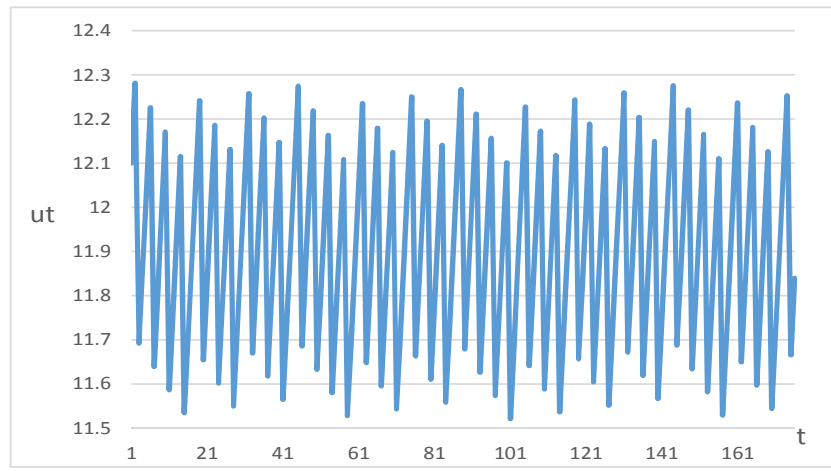

Figure 5. A simulated result of the difference equation system.

The graph exhibits more complicated behavior than two-period cycles. Hence, these estimation results suggest that the process has a non-linear phenomenon caused typically by limit cycles.

\section{Conclusions}

In this study, we investigated the non-linearity of the Japanese business cycle based on the theoretical concepts of a limit cycle. To analyze the time series of capital stock and GDP simultaneously based on the theoretical causal relationships predicted by a limit cycle, we applied the TAR model to the capital coefficient and investigated fluctuations in the coefficient that are concurrent to the underlying oscillation of the limit cycle.

The estimation results indicate that these series are subject to a three-regime TAR model and that the middle regime has divergence and the outside regimes have convergence. These results are consistent with the features of a limit cycle, suggesting that the process has a non-linear phenomenon caused typically by limit cycles.

\section{Acknowledgement}

The authors wish to thank seminar participants in the Hayama meeting spring 2015, and particularly Toichiro Asada, Akio Matsumoto, Harutaka Takahashi, and Masatoshi Yoshida for their helpful comments. The authors also thank anonymous referees whose comments greatly helped to improve the paper. All remaining errors are our own responsibility. The paper is dedicated to Professor Toshikazu Ito on the occasion of his retirement at Ryukoku University.

\section{Appendix A}

\section{A-1. Jacobian matrix of the dynamic system}

To investigate the uniqueness and stability of the fixed points $\left(Y^{*}, K^{*}\right)$, by applying Taylor expansion on equations (3) and (4) and linearizing them at the fixed point, we obtain a Jacobian matrix as follows:

$$
J^{*}=\left[\begin{array}{cc}
\alpha\left(I_{Y}^{*}-S_{Y}^{*}\right) & \alpha\left(I_{K}^{*}-S_{Y}^{*}\right) \\
I_{Y}^{*} & I_{K}^{*}-\delta
\end{array}\right],
$$

where $J^{*}=J\left(Y^{*}, K^{*}\right), I_{Y}^{*}=I_{Y}\left(Y^{*}, K^{*}\right)$, and $I_{K}^{*}, S_{Y}^{*}, S_{K}^{*}$ are shown in the same manner, too.

The trace and determinants of the matrix are

$$
\begin{gathered}
\operatorname{tr} J^{*}=\alpha\left(I_{Y}^{*}-S_{Y}^{*}\right)+\left(I_{K}^{*}-\delta\right), \\
\operatorname{det} J^{*}=\alpha\left(I_{Y}^{*}-S_{Y}^{*}\right)\left(I_{K}^{*}-\delta\right)-\alpha\left(I_{K}^{*}-S_{K}^{*}\right) I_{Y}^{*} .
\end{gathered}
$$

If we indicate the characteristic equation as

$$
\lambda^{2}-\operatorname{tr} J^{*} \lambda+\operatorname{det} J^{*}=0,
$$

we get two characteristic roots associated with equation (A4)

$$
\lambda_{1}, \lambda_{2}=\frac{t r J^{*} \pm \sqrt{\left(t r J^{*}\right)^{2}-4 \operatorname{det} J^{*}}}{2} .
$$

From the relationship of the coefficients and solution in the equation, it can also be shown that $\operatorname{trJ} J^{*}=\lambda_{1}+\lambda_{2}, \operatorname{det} J^{*}=\lambda_{1} \cdot \lambda_{2}$.

\section{A-2. Limit cycles in the Kaldor model}

We can find the existence of limit cycles in the system of the equation by applying the Poincare-Bendixson theorem. Here, if the solution locus of the system is characterized by limit cycles, the level of national income and capital stock are continuously fluctuating, implying the existence of long-lasting business cycles.

Consider a system of differential equations on $R^{2}$ as follows: 


$$
\begin{aligned}
& \dot{x_{1}}=f\left(x_{1}, x_{2}\right), \\
& \dot{x_{2}}=g\left(x_{1}, x_{2}\right) .
\end{aligned}
$$

Let the trajectory starting from the initial condition $x^{0}=\left(x_{1}^{0}, x_{2}^{0}\right)$ be $\phi_{t}\left(x^{0}\right)$. A limit set appears in the Poincare-Bendixson theorem according to the following definition.

DEFINITION (Limit Set): The $\omega$ limit set of a point $x \in W$ is defined as all the points $l \in W$ such that for the sequence $t_{i} \rightarrow \infty, \lim _{i \rightarrow \infty} \phi_{t_{i}}(x)=l$. The $\alpha$ limit set is also defined in the same way for the sequence $t_{i} \rightarrow-\infty$.

Generally speaking, there are three kinds of limit sets in the model, namely a stable fixed point, a closed orbit, and a saddle loop, which consists of a single saddle point and two unstable fixed points. If the uniqueness condition of the solution is assured, the limit set is either a fixed point or a closed orbit. If the $\omega$ limit set is an isolated closed orbit, it is called a stable limit cycle. If the $\alpha$ limit set is an isolated closed orbit, it is called an unstable limit cycle.

If we call the system of differential equations indicated by equations (A5) and (A6) $X$, its vector space notation is indicated as follows:

$$
\vec{X}=f\left(x_{1}, x_{2}\right) \partial / \partial x_{1}+g\left(x_{1}, x_{2}\right) \partial / \partial x_{2} .
$$

Domain $D$ on $R^{2}$ is diffeomorphic to a disk and assumed to be piecewise differentiable on boundary $\partial D$. Furthermore, the number of singular points in the interior of domain $D$ is assumed to be finite.

Consider the dynamic system $X$ in the neighborhood of domain $D$ and assume that $X$ is transverse in boundary $\partial D$. If we call an orbit that passes through point $P$ on the boundary of $D P \in \partial D \quad \gamma(t)=\phi_{t}(P)$, then the limit set of the orbit $\lim _{t \rightarrow \infty} \gamma(t)$ is stated as follows (Lefschetz (1962]).

Theorem (Poincare-Bendixson): A limit set of orbit $\gamma$ $\lim _{t \rightarrow \infty} \gamma(t)$ is one of the three cases shown below.

$\gamma$ reaches the singular point monotonically or by rotating around it.

$\gamma$ twines the closed orbit $S^{1}$ and a limit set $\Lambda^{+}(\gamma)=\lim _{t \rightarrow \infty} \gamma(t)$ is the closed orbit of circumference $S^{1}$.

A limit set $\Lambda^{+}(\gamma)=\lim _{t \rightarrow \infty} \gamma(t)$ is a saddle loop or sequences of saddle loops.

Proof: See Lefschetz (1962).

The intuitive explanation of the theorem and its application runs as follows. In domain $D$, vector space $\vec{X}$ points inward on boundary $\partial D$. This means that once an orbit enters into domain $D$, it will stay inside the domain thereafter.

If the fixed point is stable, the solution orbit $\gamma$ reaches the fixed point monotonically or by rotating around it (case a). If the fixed point(s) is (are) unstable, the possible limit set is only a closed orbit (case b) or a saddle loop (case c). In these cases, if the fixed point is unique, all the limit sets in the domain converge to the closed orbit, because in the case of a saddle loop, at least one more fixed point should exist.

The procedure for applying the Poincare-Bendixson theorem is as follows. First, the existence of a stable point with saddle point stability is excluded. A condition that satisfies the unstable nature of the stable point is then examined. Finally, the phase of the system of differential equations and existence of compact and invariable set " $\mathrm{D}$ " are examined.

Exclusion of saddle point stability

To exclude the saddle point stability of the stable point, $\operatorname{det} J^{*}=\lambda_{1} \cdot \lambda_{2}>0$ in equation (A1) is needed. Therefore,

$$
\operatorname{det} J^{*}=\alpha\left(I_{Y}^{*}-S_{Y}^{*}\right)\left(I_{K}^{*}-\delta\right)-\alpha\left(I_{K}^{*}-S_{K}^{*}\right) I_{Y}^{*}>0
$$

is assumed here.

Instability of the stable point For the instability of the stable point, the trace and determinant of the equation must be positive, i.e. $\operatorname{tr} J^{*}=\lambda_{1}+\lambda_{2}>0, \operatorname{det} J^{*}=\lambda_{1} \cdot \lambda_{2}>0$ :

$$
\alpha\left(I_{Y}^{*}-S_{Y}^{*}\right)>-\left(I_{K}^{*}-\delta\right) .
$$

From the assumption mentioned above, $I_{K}<0$, which makes the right-hand side of the equation positive. Therefore, the national income-induced investment effects must be considerably higher.

The assumption on the non-linear investment function stated above means that $I_{Y}$ decreases as $Y$ moves away from the stable point, which makes $\left(I_{Y}-S_{Y}\right)$ negative when national income is small or large enough.

Existence of the compact $D$ set

In the Kaldor model, a set $D=\left\{(Y, K) \mid 0 \leq Y \leq Y_{1}, 0 \leq K \leq K_{1}\right\}$ is compact because of the assumptions of the model and the vector space is inward pointed at the bound of the set. In this manner, a limit cycle may exist.

Appendix B. Dynamic phase and the vector space of the dynamic system

To examine the phase of the system, we put $\dot{K}=0$ in equation (1) and differentiate to obtain

$$
\frac{d K}{d Y_{\text {at } \dot{K}=0}}=-\frac{I_{Y}}{I_{K}-\delta}>0 .
$$

Equation (A9) implies that the $\dot{K}=0$ locus is upward sloping. Similarly, by putting $\dot{Y}=0$ in equation (2) and differentiating, we obtain 


$$
\frac{d K}{d Y}_{a t \dot{Y}=0}=-\frac{I_{Y}-S_{Y}}{I_{K}-S_{K}} .
$$

Equation (A10) takes both a positive and a negative sign. However, the locus has a positive slope near to the stationary point and a negative slope far from the stationary point.

\section{References}

[1] Agliari, A., R. Dieci, and L. Gardini, (2007), "Homoclinic Tangles in a Kaldor-like Business Cycle Model," Journal of Economic Behavior and Organization, Vol. 62, pp. 324-347.

[2] Bec, F., M. Ben Salem, and M. Carrasco, (2004) "Tests for Unit-root Versus Threshold Specification with an Application to the Purchasing Power Parity Relationship," Journal of Business and Economic Statistics, Vol. 22, pp. 382-395.

[3] Bischi, G. I., R. Dieci, G. Rodano, and E. Saltari, (2001), "Multiple Attractors and Global Bifurcations in a Kaldor-type Business Cycle Model," Journal of Evolutionary Economics, Vol. 11, pp. 527-554.

[4] Chang, W. W. and D. J. Smith, (1971), "The Existence and Persistence of Cycles in a Non-linear Model: Kaldor's 1940 Model Re-examined," Review of Economic Studies, Vol. 38, pp. 37-44.

[5] Hansen, B. E., (1999), “Testing for Linearity," Journal of Economic Surveys, Vol. 13, No. 5, pp. 551-576.

[6] Kaldor, N., (1940), "A Model of the Trade Cycle," Economic Journal, Vol. 50, pp. 78-90.

[7] Kapetanios, G. and Y. Shin, (2006) "Unit Root Tests in Three-regime SETAR Models,” Econometrics Journal, Vol. 9, pp. 252-278.

[8] Koop, G. and S. M. Potter, (1999), "Bays Factors and Nonlinearity: Evidence from Econometric Time Series," Journal of Econometrics, Vol. 88, pp. 251-281.
[9] Kraeger, H. and P. Kugler, (1993), "Non-linearities in Foreign Exchange Market: A Different Perspective," Journal of International Money and Finance, Vol. 12, pp. 195-208.

[10] Lefschetz, S., (1962), Differential Equations: Geometric Theory 2nd ed., Interscience Publishers.

[11] Lorentz, H. W., (1993), Non-linear Dynamical Economics and Chaotic Motion, Springer-Verlag.

[12] Maki, D., (2009) "Tests for a Unit Root using Three-regime TAR Models: Power Comparison and some Applications, Econometric Reviews, Vol. 28, pp. 335-363.

[13] Maki, D., and S. Kitasaka, (2014), "Residual-based Tests for Cointegration with Three-regime TAR Adjustment," Empirical Economics, forthcoming.

[14] Nishigaki Y., Y. Ikeda, and M. Satake, (2007), "A Non-Linear Approach to the Japanese Business Cycles," Global Business and Finance Review, Vol. 12, No. 3, pp. 41-50.

[15] Park, J. Y., and M. Shintani, (2014) "Testing for a Unit Root Test against Transitional Autoregressive Models," International Economic Review, forthcoming.

[16] Potter, S. M., (1995), “A Non-linear Approach to U.S. GDP," Journal of Applied Econometrics, Vol. 10, pp. 109-125.

[17] Sarantis, N., (1999), "Modeling Non-linearities in Real Effective Exchange Rates," Journal of International Money and Finance, Vol. 18, pp. 27-45.

[18] Satake, M., Maki, D., and Y. Nishigaki, (2009) "Limit Cycles in Japanese Macroeconomic Data: Policy Implications from the View of Business Cycles," International Journal of Economic Policy Studies, Vol. 4, pp. 37-54.

[19] Tong, H., (1983), Threshold Models in Non-linear Time Series Analysis: Lecture Notes in Statistic 21, Springer-Verlag. and K. S. Lim, (1980), "Threshold Autoregression, Limit Cycles and Cyclical Data," Journal of the Royal Statistical Society, Series B (Methodological), Vol. 42, No. 3, pp. 245-292.

[20] Varian, H. R. (1979), "Catastrophe Theory and the Business Cycle," Economic Inquiry, Vol. 17, pp. 14-28. 